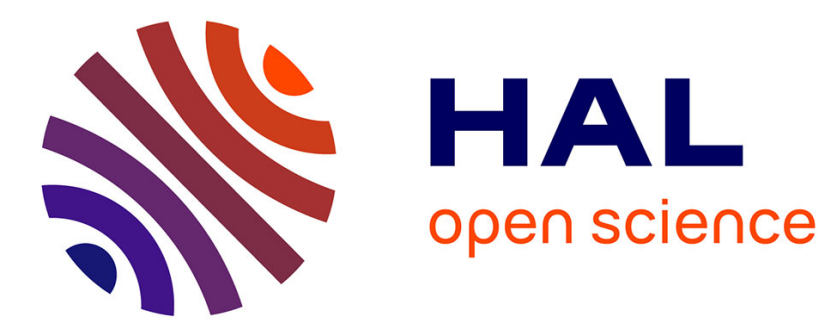

\title{
Une ardente alchimie ou l'immortalité par les cuisines
} Vincent Durand-Dastès

\section{To cite this version:}

Vincent Durand-Dastès. Une ardente alchimie ou l'immortalité par les cuisines. Marie Bizais-Lillig, Sandra Schaal. Éducations sentimentales en contextes orientaux, Presses Universitaires de Strasbourg, pp.355-376, 2019, Études orientales, slaves et néo-helléniques, 978-2-86820-992-4. halshs-02108635

\section{HAL Id: halshs-02108635 \\ https://shs.hal.science/halshs-02108635}

Submitted on 24 Apr 2019

HAL is a multi-disciplinary open access archive for the deposit and dissemination of scientific research documents, whether they are published or not. The documents may come from teaching and research institutions in France or abroad, or from public or private research centers.
L'archive ouverte pluridisciplinaire HAL, est destinée au dépôt et à la diffusion de documents scientifiques de niveau recherche, publiés ou non, émanant des établissements d'enseignement et de recherche français ou étrangers, des laboratoires publics ou privés. 


\section{Une ardente alchimie ou l'immortalité par les cuisines. Vincent DURAND-DASTÈs}

Le statut des femmes dans les religions chinoises est, comme dans bien des systèmes religieux du monde, marqué par l'ambiguïté. Les femmes sont frappées d'infériorité à bien des égards, car considérées comme pouvant être rituellement impures et admises en portion relativement congrue dans la plupart des clergés. Pourtant, les religions chinoises comptent un nombre appréciable de déesses, qui occupent des positions parfois tout à fait éminentes, et toutes sortes de «spécialistes religieux» de sexe féminin, tout à fait indispensables à la vie religieuse des communautés, tels les medium et exorcistes. Certaines de ces prêtresses dirigèrent d'importants mouvements religieux, qui, s'étendant parfois à plusieurs comtés ou provinces, inquiétèrent sérieusement les autorités. Par ailleurs, en Chine comme en bien d'autres lieux, les fidèles de sexe féminin comptaient pour une grande partie de la «clientèle» des monastères et lieux de culte. Pourtant, une morale officielle prônant la stricte séparation des sexes tenta à maintes reprises d'écarter les femmes de l'activité religieuse, du moins lorsque celle-ci impliquait pour elles de franchir les limites de leur maisonnée ${ }^{1}$. Ecoutons par exemple ce discours tenu par le personnage d'un roman chinois du $\mathrm{XVII}^{\mathrm{e}}$ siècle à un groupe de femmes qu'il a eu la surprise de rencontrer sur le chemin d'un temple où elles comptent faire leurs dévotions :

「是誰家没禮義男子，放縱閨門婦女外游？有這等不知差婦女，借口燒香，庵觀混雜。雖 然是釋門, 清淨慈悲, 普度善男信女, 只恐藏奸導欲, 引惹市井無賴頑心。女菩薩有這善 心, 何不守婦道, 不出閏門, 在家堂焚香拜聖; 何必瞞丈夫, 信僧尼, 入寺觀, 出身露面 , 見像焚修!」《Quelle est donc cette famille dont les hommes, sans principes ni bienséance, laissent leurs femmes se promener librement? Et quelles femmes sans vergogne, qui prétextent un pèlerinage pour aller se plonger dans la promiscuité des temples ou des ermitages! Même si elles vont chez des moines bouddhistes pleins de pureté et de compassion, dévoués à sauver les fidèles des deux sexes, on peut craindre qu'elles n'éveillent les désirs cachés des débauchés de la ville. Mesdames les Bodhisattvas! Si une pieuse impulsion vous motive, que n'observez-vous les règles de votre sexe ? Demeurez plutôt dans le gynécée, à brûler de l'encens et adorer les saints devant l'autel familial ! Cela vaut-il la peine

\footnotetext{
${ }^{1}$ Voir sur ce sujet Vincent GoOsSAERT, 2008, p. 212-241.
} 
de mentir à votre mari, de vous fier aux bonzes et, en entrant dans les temples, d'exposer vos corps à la vue, rien que pour contempler les statues et brûler de l'encens ? »²

C'est à dessein que, parmi bien d'autres textes qui exprimeraient une position tout à fait analogue, nous avons cité ici un extrait romanesque. Le roman en langue vulgaire (tongsu xiaoshuo 通俗小説) chinois, genre qui devait connaitre une grande vogue à partir de la dynastie des Ming (1368-1644), ouvrit en effet dans les derniers siècles de la Chine impériale, à côté de la littérature en chinois classique, langue des belles lettres et de l'administration, un espace littéraire d'une grande richesse et complexité. S'employant à concilier culture lettrée et culture populaire, à panacher dans de mêmes récits les leçons de chacun des «trois enseignements ${ }^{3}$ », à marier descriptions poétiques, récits saisissants et dialogues pleins de verdeur, le roman en langue vulgaire apporte sur le monde réel et imaginaire des Chinois Ming et Qing (1644-1911) un éclairage bien particulier.

\section{Romans hagiographiques de la fin de l'époque impériale}

Bien qu'extérieurs aux canons des religions constituées, les romans en langue vulgaire ont eu bien souvent des thèmes religieux, qu'ils ont concouru à populariser et expliquer ${ }^{4}$. Ils se caractérisent aussi par leur grand nombre d'héroïnes, qui éclipsent souvent leurs alter ego masculins. Ils contiennent de ce fait un certain nombre d'adresses faites aux disciples femmes par des maîtres religieux, que l'on serait en peine de trouver dans la littérature canonique. C'est notamment le cas dans un sous-genre du roman, celui des hagiographies en langue vulgaire qui connut une certaine vogue entre la fin de la dynastie des Ming et le début de la dynastie des Qing. On peut dénombrer une vingtaine de textes parus entre les années 1560 et 1660 qui adoptent la forme du roman en langue vulgaire pour conter l'odyssée d'une divinité ou d'un groupe de divinités, en général au travers d'une incarnation terrestre, où le ou les héros doivent affronter force épreuves pour conquérir ou reconquérir leur statut divin ${ }^{5}$. Aux côtés de ces hagiographies romanesques, généralement courtes, on

\footnotetext{
${ }^{2}$ Qingxi daoren, Dongdu ji, chapitre 31, p. 269. Trad. Vincent DURAND-DASTÈs, 2008, p. 91. Pour une satire d'un pèlerinage féminin dans un roman du XVIIe siècle, voir les deux articles de Glen DUDBRIDGE, 1991 et 1992.
}

${ }^{3}$ Le concept de "trois enseignements" (sanjiao 三教) accordait droit de cité au confucianisme, bouddhisme et taoïsme ; il excluait les pratiques religieuses populaires et surtout toute nouvelle tentative de synthèse religieuse non sanctionnée par les autorités.

${ }^{4}$ Voir V. DURAND-DASTÈs, 2002.

${ }^{5}$ LI Fengmao, 1996 et 2003. 
doit aussi compter parmi les œuvres narratives axées autour du thème du progrès religieux des romans fleuves qui sont entre bien des choses des allégories de la quête de la perfection, tels le chef-d'œuvre de la fin du XVI siècle La Pérégrination vers l'Ouest (Xiyou ji 西遊記) ainsi que ses suites ou imitations. A partir du milieu du XVII ${ }^{\mathrm{e}}$ siècle, le centre de gravité du discours hagiographique en langue vulgaire semble s'être déplacé : on n'en voit plus guère adopter la forme du tongsu xiaoshuo. Les hagiographies semblables à celles d'époque Ming en ce qu'elles sont centrées autour d'une divinité ou d'un groupe de divinités prennent désormais le plus souvent la forme du baojuan 寶卷 ou «Rouleaux précieux », un genre prosimétrique où le récit est coupé de longs segments versifiés, destinés à être chantés lors de représentations données par des laïcs ou des prêtres ${ }^{6}$.

\section{«Qui eut cru qu'il y ait en ce monde fille au cœur si féroce !»}

Les grands personnages du taoïsme figurent en bonne place dans les romans hagiographiques des $\mathrm{XVI}^{\mathrm{e}}$ et XVII ${ }^{\mathrm{e}}$ siècles. Deux de ces romans taoïstes ont la particularité d'avoir pour sujet non un individu, mais un groupe. Le premier est le roman que WU Yuantai 吳元泰 consacra aux populaires «Huit immortels», La Pérégrination vers l'Est ${ }^{7}$; le second, anonyme, conte l'histoire des «Sept parfaits », les disciples du fondateur de l'ordre taoïste Quanzhen 全真, WANG Zhe 王喆 (1113-1170) ${ }^{8}$. Ces deux, groupes, essentiellement masculins, ont pour point commun d'admettre dans leurs rangs une femme : HE Xiangu 何仙姑 (He «l'immortelle») pour les Huit immortels, SuN Bu'er 孫 不二 (Sun « la non-duelle ») $)^{9}$ pour le groupe des Sept parfaits.

\footnotetext{
${ }^{6}$ Sur les baojuan hagiographiques en général, voir Mark BENDER, 2001. Sur les rôles religieux des personnages féminins dans les baojuan, voir Beata Grant, 1995.

${ }^{7}$ Titre complet Baxian chuchu dongyou ji 八仙出處東遊記. Ce roman a été traduit en français par Nadine Perrront, La Pérégrination vers l'Est, Gallimard, Paris, 1993.

${ }^{8}$ L'appellation collective de « Sept parfaits » désigne les premiers disciples du fondateur de l'école taoïste Quanzhen Wang Chongyang (Wang Zhe) : Ma Danyang, Tan Chuduan, Liu Chuxuan, Qiu Chuji (Qiu Changchun), Wang Chuyi, Hao Datong, et Sun Bu'er, la seule femme du groupe. Le groupe a fait l'objet d'hagiographies collectives en langue classique dès 1271 avec les Qizhen nianpu 七真年譜(Chroniques par années des Sept parfaits). Voir Pierre MARSONE, 2001, p. 97. Sur les débuts du mouvement Quanzhen, voir aussi Vincent GOOSSAERT, 2001, et Stephen ESKILDSEN, 2004.
}

${ }^{9}$ Sur Sun Bu'er dans le taoïsme Quanzhen, voir Catherine DESPEuX, 1990. 
Nous nous attarderons un instant sur la scène par laquelle, dans Les biographies des patriarches les Sept parfaits (Qiə̧hen zushi lię̧uan 七真祖師列傳) ${ }^{10}$, WANG Zhe, après avoir d'abord refusé, accepte finalement de dispenser son enseignement à SUN Bu'er, faisant d'elle sa première disciple.

La rencontre a lieu dans la première partie du récit : WANG Zhe, initié par les immortels LÜ Dongbin et ZHONGLI Quan, s'est rendu au Shandong, où il a été accueilli par un couple en quête de perfection, MA Yu et sa femme Sun, qui ont reconnu en lui un homme exceptionnel. WANG se comporte pourtant comme un maitre revêche, refusant ses enseignements et rudoyant ses deux hôtes. SuN, consciente de la brièveté de la vie humaine et soucieuse de ne pas entamer trop tard son apprentissage de la Voie, décide un beau jour de hâter les choses et de demander en face à WANG qu'il l'initie enfin. La première réaction de Wang est quelque peu réfrigérante: il doute pour commencer qu'une femme soit capable de supporter les austérités de la vie recluse. Mais, comme elle insiste avec vigueur, il concède croire à sa détermination et à la sincérité de sa requête. Aux yeux du maitre un obstacle important demeure toutefois:

《你年方二十七，八，正是青春少年，風流儀表人材，好比王母娘娘，斗牛宮內 得仙女一般。世人見了，那個不愛？都想與你如此起來，怎麼煅煉身心？就是美貌 得大病, 將你害了。上乘得功夫, 功滿要作上仙地位。恐你有些難處。依我說來, 或是做一個中乘, 或是做一個下乘。”

«Tu n'es âgée que de vingt-sept ou vingt-huit ans, et tu es vraiment dans la fleur de ta jeunesse. Par tes grâces, ta mine et ton talent, tu es tout à fait comparable aux fées du Palais Douniu, ou à la Reine-Mère d'Occident. Qui ne tomberait amoureux de toi sitôt qu'il t’aperçoit ? Si tous te considèrent ainsi, comment pourrais-tu affiner par l'alchimie ton corps et ton esprit? C'est le grand défaut de ta beauté qui te nuit! Les exercices du véhicule supérieur conduisent à la dignité d'immortel du plus haut rang. Je crains que cela ne te soit difficile. Si tu m'en crois, tu choisiras d'emprunter le véhicule médian ou le véhicule inférieur. »

\footnotetext{
${ }^{10}$ Qizhen zushi liezhuan in Quanzhen qizi quanshu, p. 157-236. Ci-après Les Sept parfaits. Seule une réédition de la fin du XIX ${ }^{\mathrm{e}}$ siècle des Sept parfaits nous a été transmise. Il existe toutefois bien des indices que ce roman ait fait partie du groupe des hagiographies Ming. Suite à sa réédition à la fin des Qing, plusieurs nouvelles versions du récit ont été rapidement composées, dans le but avoué d'actualiser et de corriger une «version ancienne » jugée obsolète. Sur ce cycle romanesque, je me permettrai de renvoyer à mon étude «A late Qing blossoming of the Seven lotus » in Liu Xun \& Vincent Goossaert (éd.), Quanzhen Daoists in Chinese Society and Culture. Pour une traduction anglaise d'une des versions de la fin du XIX siècle, voir Eva WONG, Seven taoist masters, Shambala, Boston, 1990.
} 
Et le patriarche de proposer à Sun de s'attacher à suivre plutôt des chemins de salut plus à sa portée : elle pourrait ainsi réciter avec constance les mantras et conjurations, de façon à rejoindre les immortels de seconde classe au Palais du Méridien. À défaut, les techniques psychophysiques lui permettraient sans doute, comme adepte de troisième classe, d'étendre son laps de vie et de jouir de la longévité. Si cette voie lui parait trop ardue, elle pourrait encore accéder à la quatrième classe des adeptes : ceux qui, en menant une vie vertueuse et charitable, obtiennent, au terme de celle-ci, d'accéder à une réincarnation plus avantageuse : dans son cas, il s'agirait de renaitre garçon : «Si ta résolution ne faiblit pas dans ta nouvelle existence, tu pourras alors accomplir la Voie». Mais Sun, en rien ébranlée, continue de réclamer d'être éveillée au véhicule supérieur, et repousse les offres du maitre d'emprunter les voies mineures vers le salut. Elle comprend que le moment est venu pour elle d'inscrire sa résolution dans son propre corps, puisque c'est celui-ci qui constitue le nœud du problème :

“師父，你說我的容貌不能悟上乘的功夫，這有何難？略等片時，在看我的容顏何 如。”孫氏即道廚房，將油放在鍋內，架起火來，登時把烏雲燒盡；面目放在油鍋 裏邊，著水點煉個難看的模樣；臉上刺破。走到師父面前，叫聲：“師父，弟子這 個模樣，可悟上乘否？”王祖一看，大吃一驚，見孫氏合醜鬼一般，說到：“善哉， 善哉, 世上那有這樣狠心的女子? 真乃女中丈夫！就是男子中也無這大剛大智之 人! ”遂作詩一首。詩曰：

\section{女中丈夫悟上乘, 大剛大智可修行 火煉容貌似醜鬼，功滿行完白日升。}

«Maitre, si vous dites que c'est mon joli minois qui m’empêche de m’éveiller au véhicule supérieur, où est la difficulté ? Accordez-moi un bref instant, et vous verrez à quoi ressemble mon joli minois!»

Madame SUN se rendit aux cuisines, versa de l'huile dans un chaudron, et alluma du feu : en un instant, les noires volutes de ses cheveux s'étaient consumées. Elle pencha son visage vers le chaudron d'huile bouillante où elle jeta de l'eau, se faisant par cette alchimie une physionomie difficile à contempler : son visage était constellé de brûlures. Elle revint trouver son maitre, lui disant :

«Maitre, maintenant que votre disciple a cette apparence, peut-elle être éveillée au véhicule supérieur? ? Le patriarche WANG la regarda et sursauta : Madame SUN était en tout point semblable à un hideux démon. Il s'exclama : «Bénédiction! Bénédiction! Qui eût cru qu’il y ait en ce monde fille au cœur si féroce ! C'est un vrai mâle d'entre les femmes! Encore n'y a- 
t-il guère d'hommes qui aient telle fermeté et telle sagesse!» Et il composa aussitôt ce poème, qui disait :

Un vrai mâle d'entre les femmes s'éveille au vébicule supérieur ;

C'est grande résolution, grande sapience : elle pourra pratiquer la culture de soi.

Ayant par l'alchimie du feu produit visage de démon bideux,

Quand elle aura achevé ses travaux méritoires,

C'est en plein jour qu'elle montera aux cieux!»

Littéralement subjugué par l'atroce détermination de sa disciple, Wang ravale en un instant objections et réticences : elle s'est montrée digne, non seulement de figurer parmi ses disciples, mais même de prendre place au premier rang d'entre eux, hommes ou femmes confondus. Le patriarche s'empresse d'adresser à celle qu'il rebaptise Sun «la-non-duelle » un prêche imagé, qui occupe presque toute la seconde partie de cette scène d'éveil aux principes du Dao. Wang y tient à la jeune femme un discours passionné, non sans beauté, où il emploie avec lyrisme les images décrivant les progrès de l'alchimie intérieure ou «cinabre intérieur» (neidan 內丹), autrement dit les techniques psycho-physiques et rituelles par lesquelles les adeptes taö̈stes, prenant leur propre corps comme creuset, faisaient par raffinement progressif naître et crôttre en eux l'embryon d'immortalité ${ }^{11}$. Ayant dévoilé à Sun les mystères dont il la jugeait initialement indigne, il la charge de se rendre au Henan mener la vie d'une ascète errante, pour y endurer les austérités mêmes qu'il croyait insupportables pour une femme peu de temps auparavant :

\section{孫不二拜別師父，瘋了魔了，身穿破衣，竟往河南洛陽縣。一路上討飯充飢，夜住 曉行。不日來到洛陽縣，宿住古廟裏邊，白日上街乞食，有一月天氣。街坊上做買}

\footnotetext{
${ }^{11}$ On en touvera la traduction intégrale en appendice de ce chapitre. Les termes employé par Wang Zhe dans le discours à SUN qu'on pourra y lire sont empruntés au vocabulaire, à la fois technique et très imagé, des traités de neidan : éléments microcosmiques et macrocosmiques y sont constamment mis en rapport : ainsi cœur et reins forment une paire complémentaire correspondant respectivement au mercure et au plomb, au dragon et au tigre, au ciel et à la terre. Le «manche du boisseau » désigne les trois des sept étoiles de la grande ourse dont la forme évoque un manche (les quatre autres étant comparées au récipient appelé boisseau) : la Grande ourse, siège d'un puissant pouvoir exorciste céleste, joue un rôle clef dans les rituels taoïstes. Il ne saurait être question d'expliciter ici en détail la riche imagerie mobilisée pour décrire les progrès de l'adepte dans les étapes successives de ces pratiques psychophysiques: on se bornera à dire qu'il s'agit d'un processus par lequel l'adepte, renversant le cours normal des choses, obtient, par raffinement et régressions successives, de faire croître en son corps l'embryon d'immortalité. Pour une introduction à la théorie et aux pratiques du neidan, et la différence avec l'alchimie extérieure (waidan), voir Pierre-Henry DE BRUYN, «Transformation matérielle du corps : waidan » et « Transformation spirituelle du corps : neidan », in idem, 2009, p. 129-187. On lira également avec profit le chapitre de Fabrizion Pregadio et Lower Skar, «Inner alchemy (neidan) » in Livia Kohn, ed., Daoism handbook, p. 463-497
} 
賣的人都說：“那裏來這個瘋女人? ”眾人就問孫不二：“你從那裏來的? ”孫不二並 不回言, 只管胡說亂語。看他頭臉上, 好像火燒的孫猴子。眾人再不細問。

Sun-la-non-duelle prit congé de son maître, fut saisie de folie, se vêtit de haillons, et s'en fut tout droit vers Luoyang au Henan. Elle mendiait sa pitance en chemin, cheminant tout le jour pour ne s'arrêter qu'à la nuit tombée. Avant peu, elle arriva à Luoyang, où elle prit ses quartiers dans un vieux temple. Le jour, elle mendiait dans les rues, et vécut ainsi un premier mois. Les marchands ambulants disaient d'elle: «D'où vient cette folle femme?» Ils lui demandèrent: «D’où viens-tu? » Mais Sun «la-non-duelle» ne leur répondait pas, marmonnant des paroles sans queue ni tête. Les gens qui contemplaient son visage virent qu'elle ressemblait à un SuN-le-singe qu'on aurait passé par les flammes : ils cessèrent de l'interroger ${ }^{12}$.

La substantielle leçon initiatique que l'on rencontre dans cet épisode n'est pas ce qui en fait l'originalité: un tel discours, en effet, aurait pu être adressé dans presque les mêmes termes à un disciple mâle, et, de fait, on en trouve bien d'autres occurrences dans des textes analogues ${ }^{13}$. Beaucoup plus remarquables sont les arguments qu'oppose d'abord le maître à sa disciple putative, et la façon dont elle relève le défi qui lui est posé.

Notons que WANG Zhe ne considère nullement que SUN est intrinsèquement incapable, du fait de sa féminité, d'atteindre aux stades supérieur de la perfection religieuse, ni d'affronter les rigueurs de l'ascèse qu'il promet à ses disciples : dès qu'elle lui a affirmé sa résolution, il lui dit ne pas en douter. Mais c'est en quelque sorte à son corps défendant que SUN ne pourra suivre comme il conviendrait la voie du progrès: WANG partage en cela le point de vue du sévère moraliste de La Conversion de l'Orient que nous avons cité au début de cet article : c'est avant tout le désir dont une femme laissée libre de s'exposer aux regards ne manquerait pas d'être l'objet de la part de la gent masculine, qui, menaçant son intégrité physique, la disqualifie pour suivre le chemin dangereux de l'ascèse. La réponse de SuN est aussi radicale qu'ascétique en effet. Elle détruit aussitôt par la brûlure la dangereuse beauté qui pourrait faire obstacle à son salut; c'est une fois toute possibilité de séduction détruite qu'elle pourra en prendre le chemin. S'il n'y a donc pas dans cet épisode de refus de principe de voir une femme s'élever, c'est au prix d'une mutilation presque castratrice que la

${ }^{12}$ Qizhen zushi liezhuan, in Quanzhen qizi quanshu, juan shang, p.180-182.

${ }^{13}$ Par exemple dans la Biographie de Han Xiangzi : voir Philip CLART, 2007, p. xxii.. On en trouvera un autre exemple au chapitre trois des Epées volantes, p. 302-306 
possibilité lui en est offerte: ceci n'est pas demandé à ses condisciples hommes dont l'abstinence suffira au maittre...

\section{Feu de la cuisine, feu du four alchimique}

L'acte de SuN n'est pas sans rappeler une scène de salut qui concerne justement l'autre personnage féminin appartenant au second groupe de saints taoïstes que nous avons évoqué : He Xiangu, la seule femme de la bande des Huit immortels ${ }^{14}$. Cette scène ne figure pas dans le roman consacré aux Huit immortels, la Pérégrination vers l'Est, où la conversion de la jeune femme est décrite de façon extrêmement laconique, mais dans un autre roman taoïste de la fin des Ming, Les épées volantes (Feïjian ji 飛劍記), dont le héros est un autre membre du groupe des huit, l’immortel LÜ Dongbin 呂洞賓. Tout au long de ce roman en treize chapitres qui met en scène avec autant d'humour que d'affection ce très populaire personnage, on nous montre un LÜ Dongbin bien en peine de trouver des humains assez méritants pour pouvoir recevoir le secret de l'immortalité. De guerre lasse, son propre maitre finit par lui recommander d'aller se mêler, sous l'aspect qu'il affectionne d'un mendiant pouilleux, à la foule accourue en vue de la célébration d'une grande messe taoïste jiao 醮, tenue dans la maison d'un riche fidèle. Là, dans les cuisines, vit une jeune servante du nom de HE Huiniang: celle-là a l'étoffe d'une immortelle. De fait, dès que l'immortel déguisé se présente, la jeune-fille l'accueille avec respect et lui sert à manger. Mais rien ne se produira de plus entre eux avant que n'intervienne un tiers : celui-ci est un marchand, adepte du taoïsme, qui ne rêve de plus grand bonheur que celui de rencontrer un authentique immortel. Or, en surprenant la conversation nocturne de fantômes accourus participer au jiao, il a appris la présence dans les cuisines de l'immortel déguisé. Sachant avoir affaire à LÜ Dongbin en personne, il va se jeter à ses pieds en implorant d'être sauvé. En dépit des dénégations de LÜ, le marchand insiste tant que l'immortel finit par lâcher: «Eh, marchand! Si tu veux que je te sauve, entre donc dans le four, et je te sauverai !» Dans le four des cuisines, nous précise-t-on, brûle alors un ardent brasier. Fort de sa conviction d'avoir affaire à Lü Dongbin, le marchand essaye à plusieurs reprises de se jeter dans les flammes, mais in extremis recule à chaque fois. LÜ Dongbin prend finalement la parole, mettant un terme à ses espoirs :

\footnotetext{
${ }^{14}$ Le personnage de He Xiangu a donné lieu à une riche tradition légendaire. Voir à ce sujet WU Guangzhen, 2006, p. 287-328.
} 
純陽子笑道：「神仙恁般易做。」乃云：「眼前不是成仙客, 成仙只是姓何人。」乃以手 招著何氏女, 說道：「惠娘, 我與你鑽去。」

時何氏手中拿著個笑籬, 正欲撈飯, 因純陽子一招, 即忙過來。純陽子以手挽著何氏女雙 雙進於灶中, 火筷轉盛。眾皆大驚, 哪個還敢鑽哩? 時眾人只說何氏女被火饒死, 正在㗝 歎之際, 只見呂純陽與何氏女坐在碧雲之上, 吟詩一首雲 :

直上雲端望八都,

碧雲散盡月還孤。

茫茫四海人無數,

哪個男兒是丈夫。

«Crois-tu qu’il soit si aisé de devenir immortel ? dit LÜ Dongbin en riant. L’immortel, ce ne sera visiblement pas toi ; ici, l'immortelle, c'est celle que 'HE' on appelle!» Alors, faisant signe à la fille HE, il lui dit : «Huiniang, entrons-y ensemble !». A ce moment, la fille HE tenait à la main une louche qui lui servait à puiser le riz. Mais elle accourut dès que LÜ Dongbin lui eut fait signe. Prenant la fille HE par la main, LÜ Dongbin entra avec elle dans le four, et les flammes redoublèrent d'intensité. Tous furent saisis d'effroi : qui aurait osé les suivre? Or, juste au moment où les gens se lamentaient sur le sort de la fille HE, qui avait ainsi péri brûlée, on la vit soudain, juchée sur un nuage couleur de jade bleu-vert, en compagnie de LÜ Dongbin, qui chantonnait un poème :

Tout au faite des nuages, on voit les quatre coins de l'univers;

Bientôt se dissiperont les nuées, laissant la lune dans la solitude.

Dans ce vaste monde, les hommes pullulent:

Mais qui est le vrai mâle, dans cette multitude ?15

On est frappé des points communs entre les deux histoires. HE, tout comme Sun, choisit sans hésiter d'affronter les flammes dans l'intérêt supérieur de la quête de l'immortalité. Elle entre sans hésiter dans le brasier, accordant une confiance absolue aux injonctions du maître, comme SUN lui a sacrifié son intégrité physique : toutes deux font la preuve d'une « virilité » qui est dans les deux cas l'objet d'un poème d'éloge où elles sont assimilées au zhangfu 丈夫, la figure même de la perfection masculine: on dit de SUN qu' «un vrai mâle d'entre les femmes (nü zhangfu) s'éveille au vébicule supérieur »; pour HE, littéralement, le poème final dit «quel est donc le garçon qui serait un vrai mâle? (na ge nan'er shi z̧hangfu) ${ }^{16}$ Ce n'est donc

${ }^{15}$ Feijian ji, in Ba xian quan shu, p. 363.

${ }^{16}$ Beata Grant a montré dans quelles circonstances l'épithète de « vrai mâle » da Zhangfu a pu être appliquée aux maîtres féminins du bouddhisme Chan dans la littérature hagiographique de l'école au XVIIe siècle, époque contemporaine des romans discutés ici: Beata Grant, 2008. 
pas sans humour que les deux récits font du feu de la cuisine le moyen d'affirmation de la virilité supérieure que ces deux femmes hérö̈ques ont en partage. C'est en ce lieu éminemment féminin qu'elles atteignent au sommet de leur héroïsme ${ }^{17}$.

L'hérö̈sme qu'elles manifestent semble pourtant en contradiction apparente avec le taoïsme : cette religion ne faisait-elle pas de la culture du corps, et non de sa destruction, le but de ses pratiques? Serait-ce aller trop loin que de voir dans ces scènes de destruction rédemptrice la trace d'une méfiance profondément ancrée à l'égard du corps féminin ? Là où l'adepte masculin peut directement fortifier son corps pour le mener à l'immortalité, le corps féminin, dangereusement exposé aux souillures, ne doit-il pas plutôt être détruit, ou à tout moins radicalement et brutalement transformé pour permettre à l'adepte qui l'habite d'accéder à une condition supérieure ? C'est ce que certains récits de fictions ou anecdotes laissent parfois entendre, sans le théoriser explicitement. Ainsi, un bref mais saisissant texte ce sujet figure dans le chapitre «superstitions » (mixin lei 迷信類) du recueil Qing bai leichao 清稗類鈔 (《Anecdotes classées de la dynastie des Qing»), vaste somme de littérature anecdotique publiée en 1916 par Xu Ke 徐珂 (1869-1928). Ce bref texte traite du rituel appelé « la veille du gengshen» (zuo gengshen 坐庚申) : on y dépeint d'abord un adepte laïc qui médite et récite des écritures durant les nuits portant les caractères cycliques gengshen 庚 申 (lesquelles sont particulièrement dangereuses, car ces nuits-là les esprits des «trois vers» sanchong 三蟲, qui nichent dans notre corps et méditent sa perte, montent aux cieux pour dénoncer nos fautes afin de raccourcir notre vie) : ses pratiques lui assurent un don de prescience qui lui permettra d'échapper à l'envoyé des enfers lorsque son heure aura sonné, devenant ainsi un «immortel terrestre» (dixian 地仙). Symétriquement, le texte aborde ensuite le cas de quatre jeunes adeptes féminines des mêmes pratiques. Lorsque les deux plus âgées sont mariées par leur famille, les deux plus jeunes décident, pour préserver la pureté et l'intégrité physique (bao quan qingbai z̧hi shen 保全清白之身) que leur ont permis d'obtenir leurs pratiques assidues, de se suicider par pendaison ${ }^{18}$. Le devenir

\footnotetext{
${ }^{17} \mathrm{C}$ 'est d'ailleurs encore en ce lieu que, Sun, revenue de son long périple ascétique, démontrera sa supériorité dans les arts du Dao à son mari Ma Danyang : après s'être livrés à une joute de magie où ils se sont montrés d'égale force, elle entraîne son époux aux cuisines pour prendre sans façons devant lui un bain d'huile bouillante qu'elle a préalablement chauffée dans l'immense chaudron qui servait jadis à cuire les repas des nombreux disciples de leur maître Wang Zhe. Bien qu'elle n'en ressente désormais plus le moindre dommage, son époux se garde bien de l'imiter. Voir Qizhen zushi liezhuan, p. 208-209.
}

${ }^{18} \mathrm{Xu}$ Ke, Qingbai leichao, p. 4676. 
contrasté de l'homme et des jeunes filles qui pratiquent pourtant un même rite salvifique est saisissant : là où l'homme d'âge mûr obtient le pouvoir d'échapper à la mort tout court, c'est au contraire par la mort que les jeunes filles échappent au lot commun. Suicide et pratiques d'immortalité sont ici mis clairement en balance, le premier plus que les secondes semblant convenir plus particulièrement aux femmes...

Il convient toutefois de rappeler que, dans cette histoire comme dans les romans hagiographiques évoqués plus-haut, nous sommes dans le cadre de traditions populaires réinterprétant les pratiques taoïstes, et qu'il serait aventureux de les lire comme une sorte de soubassement inconscient de la doctrine: les textes canoniques nous ont transmis leur content de traités et guides à l'intention de l'adepte féminine, et ceux-ci ne posent nullement le suicide ou l'automutilation comme condition préalable au progrès spirituel ${ }^{19}$ ! Si des rituels de l'alchimie intérieure appelés « brûler le corps » (fenshen 焚身 ou shaoshen 燒 身 ) et destinés à combattre des forces yin 陰 existent bien ${ }^{20}$, ils se déroulent à l'intérieur du corps et s'inscrivent dans un processus alchimique global visant à renforcer, certainement pas à anéantir la personne physique - fut-elle celle d'une alchimiste femme! Pourtant, les pratiques d'auto-combustion totale existent dans le paysage religieux chinois, mais là encore sans y être plus particulièrement réservées à la gent féminine. En effet, si les pratiques sacrificielles par l'intermédiaire $\mathrm{du}$ feu sont absentes des enseignements premiers du taoïsme, elles ne le sont pas de ceux des bouddhistes : depuis l'époque médiévale, les récits portant sur des auto-immolations par le feu, ou tout au moins des pratiques de sacrifices partiels par la combustion, sont légion dans la littérature hagiographique bouddhique ${ }^{21}$. Ces pratiques ont exercé leur influence bien au-delà du cercle des adeptes de la religion de l'Eveillé. Assez fortement influencé par la culture monacale bouddhique, l'ordre taoïste Quanzhen, dont les Sept parfaits figurent parmi les patriarches, était ainsi un groupe ascétique dont les adeptes, notamment lors des quêtes, s'adonnaient fréquemment à des pratiques physiques dangereuses et mutilantes ${ }^{22}$. Les Sept parfaits dépeignent à loisir les austérités que s'infligent les héros, notamment des jeûnes extrêmes. Et au-delà, comme nos

\footnotetext{
${ }^{19}$ Voir à ce sujet Catherine Despeux, 1990 et Elena Valussi, 2009.

${ }^{20}$ Voir F. Baldrian Hussein, 1984, p. 159-163.

${ }^{21}$ Voir notamment JAN Yün-hua, 1964, et plus récemment James A BENN, 2007.

${ }^{22}$ Vincent GOOSSAERT, « Starved of resources », Harvard Journal of Asiatic Studies, vol. $62 \mathrm{n}^{\circ} 1,2002, \mathrm{p}$. 77-133.
} 
deux récits le rappellent en plusieurs points, le feu auquel se livrent les deux filles héroïques est un feu de raffinement, qui cuit le corps pour l'élever à un état supérieur : le brasier de la cuisine ou la poêle à frire servent ainsi de métaphores du four alchimique, outil fondamental de l'adepte taoïste. Cet objet, intériorisé par les pratiques de l'alchimie intérieure neidan, garde une force picturale toute prête à être employée de nouveau lorsque le traité technique laisse place au discours romanesque et à sa vivante imagerie.

\section{Saints démoniaques, féminins, ou simiesques.}

Un dernier élément enfin me paraît propre à mieux nous faire comprendre une autre connotation de la défiguration par le feu de la belle Madame SuN. Dès qu'il voit son visage constellé de brûlures, son maitre constate qu'elle est désormais «en tout point semblable à un hideux démon ». Lorsque, ayant entrepris l'errance que lui a assignée son maittre, elle va de par les rues, prise de folie, les gens finissent par se détourner d'elle voyant « qu'elle ressemblait à un SuN-le-singe qu'on aurait passé par les flammes» (buoshao de Sun hoų̧i 火 燒的孫猴子). Cette dernière allusion, sans nul doute obscure à la plupart des lecteurs français d'aujourd'hui, était tout aussi certainement parfaitement transparente à la très grande majorité du public chinois du temps. Il y a bien sûr là avant tout un jeu de mot: l'héroïne possède un patronyme, Sun, qui se prononce comme le sun de «macaque ». Mais, pour cette raison précisément, ce patronyme est aussi celui d'un des plus célèbres héros du roman et de la religion chinoises: Sun Wukong, le roi des singes, disciple d'origine simiesque du moine XuANZANG des Tang (618-907) dans le roman fantastique et religieux La Pérégrination vers l'Ouest. C'est sans nul doute à lui qu'il est fait allusion dans la phrase des Sept parfaits. Car, avant de devenir le disciple d'un bonze, Sun Wukong fut un démon singe, monstre d'une grande laideur (le récit de La Pérégrination vers l'Ouest revient en maints endroits sur l'effroi qui peut saisir ceux devant qui il se montre). Mais il a aussi été....adepte taoïste, en quête des arts de l'immortalité. Les premiers chapitres de la Pérégrination vers l'Ouest sont en effet consacrés à la quête initiale du singe : né d'un œuf de pierre, le singe magicien s'est rapidement taillé un royaume montagnard où il règne, sous le nom de « roi beau macaque », sur tout un peuple simiesque. Toutefois, profondément inquiet face à la finitude de l'existence, il part bientôt à la recherche d'un maître d'immortalité. Il le trouvera en la personne d'un maitre de Neidan vivant retiré au sein des montagnes. Or, la scène de l'apprentissage des arts de l'alchimie interne par le roi des singes, contée au chapitre deux de la Pérégrination, pourrait avoir servi de modèle à celle de l'instruction de SuN-la-nonduelle par WANG : comme à SUN Bu'er, le maître de SUN Wukong propose tout d'abord 
d'apprendre des voies subalternes ou pratiques, convenant mieux à une nature inférieure et même un peu monstrueuse, qu'elle soit simiesque... ou féminine. Comme la Non-duelle, SuN Wukong refuse avec indignation, et insiste avec obstination jusqu'à ce que le maître accepte finalement de lui livrer la quintessence du savoir ${ }^{23}$. Certes, c'est en résolvant une énigme et non en se défigurant que le singe gagne l'accès aux enseignements les plus secrets. Mais SuN Wukong est déjà monstrueux! Et, à peine sa nouvelle science alchimique apprise, il se verra voué par son maitre à un feu destructeur divin, destiné à fondre et dissoudre l'adepte démoniaque qu'il est : «Tu as dérobé le pouvoir de transformation du ciel et de la terre, tu as violé le mystère du soleil et de la lune », le préviens alors son maittre ; « quand tu auras réalisé l'élixir d'immortalité, les dieux et les démons ne sauraient le tolérer longtemps ${ }^{24} »$. Il faudra à SUN Wukong toute son audace et sa science magique pour échapper à la malédiction. Mais il n'en a pas pour autant fini avec le feu destructeur : au terme d'une querelleuse épopée au cours de laquelle il se dressera contre le Ciel il sera, finalement vaincu, livré au Vieux patriarche taoïste Laozi pour que celui-ci le fasse fondre dans son four alchimique magique: trop tard! La vitalité du singe, d'apprentissage transgressif en apprentissage transgressif, est devenue si résistante qu'il sortira encore renforcé, et non point dissout, du four alchimique. Seul le Bouddha en personne pourra le dompter et mettre sa magie au service du pèlerinage qui occupe la seconde partie du $\operatorname{roman}^{25}$.

Je ne sais si le lecteur aura l'impression à lire ces lignes que l'on rapproche exagérément l'histoire de la seule femme du groupe des Sept parfaits de la geste du roi des singes. Il me semble pourtant y avoir dans le parallélisme de l'apprentissage des deux héros plus qu'un emprunt intertextuel insignifiant. En effet, les héroïnes détentrices de savoir mystique, martial ou magique qui abondent dans la littérature en langue vulgaire, sont tout aussi célébrées qu'elles se voient attribuer un statut ambigu ${ }^{26}$ : leur féminité les fait appartenir, dans la classification cosmologique chinoise, à la sphère du yin 陰, qui est aussi celle de la nuit, de la mort, des ténèbres. Quand elles sont les héroïnes d'une histoire, elles ne sauraient être complétement dépouillées des ambiguiités attachées à leur nature. C’est en

\footnotetext{
${ }^{23}$ WU Cheng'en, Xiyou ji, chapitre 2. Voir trad. d'André LEVY, 1991, p. 29-42.

${ }^{24}$ Cité dans la traduction d d'André LEVY, 1991, p. 37.

${ }^{25}$ Wu Cheng'en, Xiyoun ji, chapitre 7. Voir trad. d'A. LEVY, 1991, p. 134-135.

${ }^{26}$ Pour une bonne analyse du statut ambigu de la sainteté féminine faite à partir de la littérature des « rouleaux précieux », voir Grant, 1995.
} 
cela qu'un personnage d'immortelle ou de magicienne peut être rapproché de celui d'un génie animal métamorphosé, comme SUN Wukong ou d'aucuns esprits ophidiens ou vulpins.

En choisissant volontiers des personnages féminins comme maittres des arts de la guerre ou de la religion, le roman en langue vulgaire inverse en quelque sorte l'ordre « normal» de la société : ce faisant, il ne peut guère les décrire que comme atypiques ou excentriques, sinon démoniaques. C'est précisément ce qui me semble être à l'œuvre dans les Sept parfaits. Sun la Non-duelle, en tant que personnage historique de l'école Quanzhen, figurait en toute légitimité dans la liste de ses figures tutélaires. Mais elle y occupait une place modeste : il faudra la liberté et le goût de la transgression du roman en langue vulgaire pour que la situation soit renversée : les Sept parfaits font de Sun la toute première disciple de WANG ${ }^{27}$, bousculant l'ordre canonique qui lui préférait son mari. De la même façon, l'auteur des Epées volantes faisait de HE Xiangu, initialement une discrète présence féminine dans le groupe des Huit immortels, le seul être mortel sauvé par le puissant LÜ Dongbin.

Il n'est dès lors pas étonnant de constater quel mode particulier de sainteté est prêté par le récit à SuN-la-non-duelle: excès, saleté, folie, errance, elle appartient sans nul doute à la cohorte étrange des «saints crasseux », qui abondent dans l'hagiographie populaire, tel le bouddhiste Ji-le-fou, lui aussi présentant des affinités avec la gent simiesque dont il affectionne la compagnie. Femme sainte, SuN-la-non-duelle ne saurait pas plus être sainte ordinaire qu'elle n'est femme ordinaire. C'est dans le registre de la sainte folie que la terrible scène de la défiguration trouve peut-être une ultime justification : alors que l'intégrité du corps est une des obsessions du taoïsme, le thème de la laideur protectrice, souvent associée à la folie, qui détourne de vous l'attention d'un monde qui finirait par vous entraîner à sa suite à votre perte, n'est-il pas un motif connu des textes taoïstes depuis les magnifiques CEuvres de Maître Zhuang ${ }^{28}$ ?

\section{Eléments bibliographiques}

Baldrian-Hussein, Farzeen, Procédés secrets du joyau magique: traité d'alchimie taoïste du XIe siècle, Paris : Les Deux Océans, 1984.

\footnotetext{
${ }^{27}$ Le romancier poussera son intérêt pour les disciples féminines en accordant à QIU Changchun, un patriarche de seconde génération, une disciple parfaitement inconnue des sources canoniques. Voir mon article à paraître, «A late blossoming of the Seven Lotus ».
}

${ }^{28}$ Voir la traduction de Jean LÉVI, Euvres de maître Tchouang, Encyclopédie des Nuisances, Paris, 2006. 
BENDER Mark, «A description of jiangïng (telling scriptures) services in Jinjiang, China », Asian Folklore Studies, vol. 60, 2001, p. 101-133.

BENN James A, Burning for the Buddha: Self-Immolation in Chinese Buddhism, University of Hawai'I press, Honolulu, 2007.

ClART Philip, «Translator's introduction », in YANG Erzeng, The Story of Han Xiangzi: the alchemical adventures of a Daoist immortal, The University of Washington press, Seattle, London, 2007.

DE BRUYN Pierre-Henry, Le taoïme : chemins de découvertes, CNRS éditions, Paris, 2009

DeNG Zhimo 鄧志謨. «Feijian ji 飛劍記》, in Hou Zhongyi 侯忠义 (éd.), Mingdai xiaoshuo jikan 明代小说辑刊, série 1, vol. 4, Ba Shu shushe, Chengdu, 1993.

DESPEuX Catherine, Immortelles de la Chine ancienne : taoïsme et alchimie féminine, Pardès, Puiseaux, 1990.

Despeux Catherine \& KoHn Livia, Women in Taoism, Three Pines Press, Cambridge, 2003.

DUdBRIDGE Glen, « A Pilgrimage in Seventeenth century Fiction: T'ai Shan and the Hsing-shib yin yüan chuan », T'oung Pao, n 77, 1991, p 226-252.

DUdBridge Glen, « Women Pilgrims to T’ai Shan: Some Pages from a Seventeenth century Novel », in NAQUIN Suzan \& YÜ Chün-fang, Pilgrims and Sacred Sites in China, University of California Press, Berkeley, 1992, p. 39-64.

DURAND-DASTĖs Vincent, «Prodiges ambigus : les récits non-canoniques sur le surnaturel, entre histoire religieuse, histoire littéraire et anthropologie », Revue bibliographique de sinologie, 2002, p. $317-$ 343.

DURAND-DASTÈS Vincent, La Conversion de l'Orient : un périple didactique de Bodhidharma dans un roman chinois en langue vulgaire du XVIIe siècle, Institut Belge des Hautes Etudes Chinoises, Bruxelles, 2008. DURAND-DASTĖs Vincent, «A late Qing Blossoming of the Seven Lotus: Hagiographic Novels about the Qizhen 七真», in LiU Xun \& GoOssaERT Vincent (éds.), Quanzhen Daoists in Chinese Society and Culture, 1500-2010, Berkeley, Institute of East Asian Studies, University of California, 2013, (China Research Monographs, 70), p. 78-112.

ESKILDSEN Stephen, The teachings and practices of the early Quanzhen Taoist masters, SUNY press, Albany, 2004.

GOOSSAERT Vincent, «The invention of an order: collective identity in Thirteenth-century Quanzhen Taoism », Journal of Chinese Religions, n² 29, 2001, p. 111-138.

GOOsSAERT Vincent, «Irrepressible Female Piety: Late Imperial Bans on Women Visiting

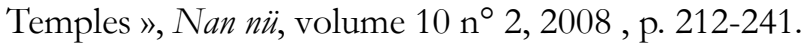

GRANT, Beata, « Patterns of female religious experience in Qing dynasty popular literature », Journal of Chinese Religions, vol. 23, 1995 p. 29-58.

GRANT, Beata « Da Zhangfu: the Gendered Rhetoric of Heroism and Equality in SeventeenthCentury Chan Buddhist Discourse Records », Nan Nü 10 (2008) p. 177-211.

JAN Yün-hua, «Buddhist self-immolation in medieval China », History of religions, vol. 4 n², 1964, p. 243-268. 
LÉVI Jean (trad.), Euvres de maître Tchouang, L’encyclopédie des nuisances, Paris, 2006.

LÉvY André (trad.), La Pérégrination vers l'Ouest (Xiyou ji), Gallimard« La Pléiade », Paris, 2 volumes, 1991.

LI Fengmao 李豐杛, Xu Xun yu sa Shoujian : Deng Zhimo daojiao xiaoshuo yanjü 許遜與薩守堅鄧志 謨道教小説研究 (XU Xun et SA Shoujian : études sur les romans taoïstes de DENG Zhimo), Taiwan xuesheng shuju, Taipei, 1996.

LI Fengmao 李豐柕, «Chushen yu xiuxing: Mingdai xiaoshuo zhefan xushu moshi de xingcheng jiqi zongjiao yiyi - yi Shuihuzhuan, Xiyouji wei zhu»出身與修行 : 明代小説謫凡敍述模式的 形成及其宗教意識- 以水滸傳、西遊記爲主 (Venue au monde et quête de la perfection : la formation du modèle narratif du bannissement dans le monde profane dans les romans Ming et sa signification religieuse - à partir essentiellement de 'Au bord de l'eau' et de la 'Pérégrination vers l'Ouest'», Guowen xuezhi 國文學誌, vol. 7, déc. 2003, p. 85-113.

MARSONE Pierre, «Accounts of. the foundation of the Quanzhen movement: a hagiographic treatment of history », Journal of Chinese Religions, $\mathrm{n}^{\circ}$ 29, 2001, p. 95-110.

Perront Nadine (trad.), La Pérégrination vers l'Est, Gallimard, Paris, 1993.

Pregadio, Fabrizio, Skar, Lowell «Inner alchemy (neidan)» in Livia Kohn, ed., Daoism bandbook, Leiden: Brill, 2000, p. 463-497.

QINGXI DAOREN 清溪道人, «Dongdu ji 東度記», in HOU Zhongyi 侯忠义 (éd.), Mingdai xiaoshuo jikean 明代小说辑刊, série 1, vol. 3, Ba Shu shushe, Chengdu, 1993.

Qizhen zushi liezhuan 七真祖師列傳 (Biographies des patriarches les Septparfaits), in QI Shoucheng 齊守成, ZHANG Ying 張穎, CHEN Su 陳速 et al., (éds.), Quan₹hen qizi quanshu 全真七子全書 (Anthologie intégrale des sept maitre du Quanzhen.), Chunfeng wenyi chubanshe, Shenyang, 1989, p.157-236.

SUN Xun 孫遜, Zhongguo gudai xiaoshuo yu zongjiao 中國古代小説與宗教 (Roman chinois ancien et religion), Fudan daxue, Shanghai, 2000.

VAlussi, Elena, "Blood, Tigers, Dragons: The Physiology of Transcendence for Women”, Asian Medicine 4 (2009), p. 46-85

WONG Eva (éd. \& trad.), Seven taoist masters: a folk novel of China, Shambala, Boston, 1990.

Wu Cheng'en 吳承恩, Xiyou ji 西遊記 (La Pérégrination vers l'Ouest), Renmin wenxue chubanshe, Beijing, 1980.

WU Guangzheng 吴光正, Baxian gushi xitong kaolun: neidandao zongjiao shenbua de jiangou ji qi liubian 八仙故事系统考论 内丹道宗教神话的建构及其流变 (Recherches et discussions sur le cycle narratif des Huit immortels : la construction et la circulation d'une mythologie de la religion Neidan.), Zhonghua Shuju, Beijing, 2006. 
WU Yuantai, «Baxian chuchu dongyou ji »八仙出處東遊記(La Pérégrination vers l'Est ou l'origine des Huit immortels.), in Hou Zhongyi 侯忠义 (éd.), Mingdai xiaoshno jikan 明代小说辑

刊 (Anthologie des romans des Ming), série 3 vol. 4, Ba Shu Shushe, Chengdu, 1999, p. 519-673.

Xu Ke 徐珂, Qingbai leichao 清稗類鈔 (《Anecdotes classées de la dynastie des Qing »),

Beijing : Zhonghua shuju, 1984, 13 volumes (éd. originale Shangwu yinshuguan 商務印書 館, 1916).

\section{Annexe : L'initiation de Sun « la-non-duelle », traduction intégrale}

[Qizhen zushi liezhuan, in Quanzhen qizi quanshu, juan shang, p.180-182.]

Un jour, MA Yu s'était rendu à la capitale du district. Madame SUN se disait in petto : «J'ai fait la rencontre d'un homme aussi éminent et je ne cherche pas à ce qu'il m'enseigne la Voie : qu'attends-je pour le faire? Si je laisse s'écouler le temps à agiter en vain mon esprit, lorsqu'arrivera le démon de l'impermanence, que me restera-t-il à faire ?» Voyant son maître assis, seul, dans sa hutte, à faire ses exercices, elle y entra et se prosterna devant lui, disant : «Maitre, votre disciple est résolue à ne plus demeurer dans le monde. Maitre, ayez de la compassion pour moi, et transmettez moi les arts du raffinement alchimique de soi!» Le patriarche WANG poussa un hurlement: «Nuisance du destin! Qu'oses-tu t’imaginer? Tu m'interroges sur les arts alchimiques, mais comment pourrais-tu supporter de dormir la nuit dans les fours à briques en ruines, ou sous les avancées des toits, ou sous les arbres en plein vent ? De te vêtir de haillons, et de ne plus parler à quiconque ? A la saison froide, de geler sans regrets? De telles souffrances, comment pourrais-tu les endurer?» Madame SUN répondit: «Ma résolution ne finira qu'avec moi : je suis tout à fait décidée à prendre ce chemin.» Le patriarche WANG répondit: «Je te crois sincère; mais un défaut majeur t'empêche de suivre ce chemin.»

- Quel est ce défaut ? demanda Madame SuN.

Le patriarche répondit : «Tu n’es âgée que de vingt-sept ou vingt-huit ans, et tu es vraiment dans la fleur de ta jeunesse. Par tes grâces, ta mine et ton talent, tu es tout à fait comparable aux fées du Palais Douniu, ou à la Reine-Mère d'Occident. Qui ne tomberait amoureux de toi sitôt qu'il t'aperçoit? Si tous te considèrent ainsi, comment pourrais-tu affiner par l'alchimie ton corps et ton esprit? C'est le grand défaut de ta beauté qui te nuit! Les exercices du véhicule supérieur conduisent à la dignité d'immortel du plus haut rang. Je crains que cela ne te soit difficile. Si tu m'en crois, tu choisiras d'emprunter le véhicule médian ou le véhicule inférieur. »

-Quel est ce véhicule inférieur? 
«Récite avec constance les mantras et les conjurations, et, ta tâche accomplie, tu prendras place parmi les immortels de deuxième classe du Palais du Méridien. Conserve avec constance le souffle primordial, et, ta tâche accomplie, tu appartiendras à la troisième classe, celle qui demeure de longues années dans le monde et vit un peu plus longtemps. Si tu n'arrives pas à entrer dans la troisième classe, il y a encore la quatrième : fixe avec constance ton esprit sur les pensées correctes, sois secourable envers autrui, soulage les tourments et les misères, fais bâtir des ponts et réparer des routes, alors, ta tâche accomplie, tu seras à ta mort réincarnée en garçon, et, si ta résolution ne faiblit pas dans ta nouvelle existence, tu pourras alors accomplir la Voie ». Madame SUN répondit : «Votre disciple s'est éveillée au véhicule supérieur, comment pourrait-elle envisager les véhicules médians ou inférieurs?»

«Je viens de te le dire », dit le patriarche WANG : «Comment avec un si joli minois pourraistu t'éveiller au véhicule supérieur? Contente-toi d’un véhicule inférieur, voilà tout!» Madame SUN répondit :

«Maitre, si vous dites que c'est mon joli minois qui m'empêche de m'éveiller au véhicule supérieur, où est la difficulté ? Accordez-moi un bref instant, et vous verrez à quoi ressemble mon joli minois !»

Madame Sun se rendit aux cuisines, versa de l'huile dans un chaudron, et alluma du feu : en un instant, les noires volutes de ses cheveux s'étaient consumées. Elle pencha son visage vers le chaudron d'huile bouillante où elle jeta de l'eau, se faisant par cette alchimie une physionomie difficile à contempler : son visage était constellé de brûlures. Elle revint trouver son maitre, lui disant :

«Maître, maintenant que votre disciple a cette apparence, peut-elle être éveillée au véhicule supérieur? ? Le patriarche WANG la regarda et sursauta : Madame SUN était en tout point semblable à un hideux démon. Il s'exclama : «Bénédiction! Bénédiction! Qui eût cru qu'il y ait en ce monde fille au cœur si féroce ! C'est un vrai mâle d'entre les femmes! Encore n'y at-il guère d'hommes qui aient telle fermeté et telle sagesse!» Et il composa aussitôt ce poème, qui disait :

Un vrai mâle d'entre les femmes s'éveille au vébicule supérieur;

C'est grande résolution, grande sapience : elle pourra pratiquer la culture de soi.

Ayant par l'alchimie du feu produit visage de démon bideux,

Quand elle aura achevé ses travaux méritoires,

C'est en plein jour qu'elle montera aux cieux!»

Le patriarche WANG ne cessait de faire son éloge, s'écriant: «Tu es vraiment ma disciple. Relève toi, et écoute mes instructions : avec ta résolution exceptionnelle, quel souci pourrait t'empêcher de rejoindre les rangs des immortels éminents ? Quand moi, ton maittre, ai reçu l'ordre d'aller recruter des disciples au Shandong, on m'a dit que je prendrai toutes sortes de disciples, hommes et femmes. Tu es assurément la toute première d'entre eux, assurée de t'élever au plus tôt vers les contrées célestes. Si tu vas au Palais pourpre de l'étang de jaspe ou 
à la Salle des nuées divines, on y inscrira ton nom. Ecoute-moi, ton maitre, te transmettre la Voie : il suffira que tu inverses cœur et reins, que tu saisisses et retournes le manche du boisseau, que tu enclenches la révolution des étoiles célestes, alors ta nature profonde et ton existence fondamentale seront ensemble cultivées, tu y parviendras! A la seconde et troisième phase, garde- toi de laisser naittre les pensées ; il faut pratiquer la diminution au fur et à mesure qu'elles naissent. Au fil des jours et de la durée, tout naturellement il ne demeurera plus rien. Dans ta pratique quotidienne, ce sera comme si tu t'ouvrais les entrailles à l'aide d'une épée, tu domineras le ressort céleste, et occiras le ressort terrestre. Lorsque périt le cœur-esprit d'un être terrestre, il y a un immortel de plus là-haut. Car quand le cœuresprit meurt, alors tout naturellement les quatre membres et le corps obtiennent de vivre. Entrave le cheval des pensées, maintiens fermement le singe de l'esprit, et tu seras divine! Quand tes exercices seront achevés, tes pratiques accomplies, tu obtiendras de t'élever jusqu'au séjour céleste : au-delà des nuages des neuf empyrées tu chevaucheras le phénix auspicieux. Ces pratiques quotidiennes, grave les bien dans ta mémoire, et garde-toi de les révéler. A présent, rends-toi dans le district de Luoyang - dans la préfecture du Henan -, car là plusieurs personnes ont une affinité karmique avec toi. Tu y feindras la folie, la faim te creusera le ventre, tu iras de porte en porte mendier ta pitance. Quand tu t'arrêteras pour la nuit, ce sera sous les avancées des toitures ou dans les fours à briques en ruines. Quand tes exercices seront achevés, tes pratiques accomplies, alors un édit de cinabre te sera adressé29, et alors seulement ta tâche sera achevée. Moi ton maitre, je vais te donner un nom religieux : on t'appellera SUN-la-non-duelle, SUN Bu'er! Va-t'en vite à présent!» SUN se prosterna et dit : « Puisqu'aujourd'hui maitre et disciple vont se séparer, quand nous reverrons-nous ? » Le patriarche WANG répondit : «Lorsque tu auras suivi jusqu’à son terme la Grande Voie, que tu auras encore accompli huit cent actions méritoires extérieures, quand tu seras finalement retournée en ta maison pour amener à l'éveil ton maitre et mari, alors toi et moi nous rencontrerons au Palais des divines nuées.»

SuN-la-non-duelle prit congé de son maitre, fut saisie de folie, se vêtit de haillons, et s'en fut tout droit vers Luoyang au Henan. Elle mendiait sa pitance en chemin, cheminant tout le jour pour ne s'arrêter qu'à la nuit tombée. Avant peu, elle arriva à Luoyang, où elle prit ses quartiers dans un vieux temple. Le jour, elle mendiait dans les rues, et vécut ainsi un premier mois. Les marchands ambulants disaient d'elle: «D'où vient cette folle femme?» Ils lui demandèrent: «D’où viens-tu?» Mais SuN-la-non-duelle ne leur répondait pas, marmonnant des paroles sans queue ni tête. Les gens qui contemplaient son visage virent qu'elle ressemblait à un SuN-le-singe qu'on aurait passé par les flammes : ils cessèrent de l'interroger.

\footnotetext{
${ }^{29}$ « édit de cinabre » : comprendre un avis de nomination dans les rangs des immortels et une invitation à venir résider au ciel.
} 
一日, 馬玉上縣去了。孫夫人暗思曰：“遇著這樣高人還不求道, 更待何時? 枉鬧 精神, 遷延歲月, 無常到了, 那時怎麼辦? ”見師父獨坐在茅庵用功, 進得門來, 倒身下拜, 口稱：“師父弟子實實不願在世上了。師父將修煉功夫傳於弟子, 望師 父慈悲慈悲! ”王祖大喝一聲, 道 : “莘障! 你真乃妄想。第問修煉功夫, 你焉能做 得到晚間宿破瓦宰中? 或宿廊檐底下, 或宿招風樹下, 身穿破爛衣衫, 並不與人答 話。過了寒冬, 凍死不悔。如此苦楚, 想你豈能受得? ”孫夫人道：“弟子一死方 休, 定然要去。”王祖道: “我看你是個真心。只有一件大病, 恐不能去。”孫夫人 道：“那一件大病? ”王祖道：“你年方二十七, 八, 正是青春少年, 風流儀表人 材, 好比王母娘娘, 斗牛宮內得仙女一般。世人見了, 那個不愛? 都想與你如此起 來, 怎麼煅煉身心? 就是美貌得大病, 將你害了。上乘得功夫, 功滿要作上仙地 位。恐你有些難處。依我說來, 或是做一個中乘, 或是做一個下乘。”孫夫人道 : “中程怎麼? 下乘怎麼? ”王祖道：“常常的念真言咒語, 行滿得做南宮二等列仙。 常常保守元氣, 功滿得做三等, 在世長年, 多活幾日。三等再若不行, 還有四等 : 常抱正念, 與人方便, 救苦救難, 修橋補路, 功滿之日, 死後轉女為難, 來生道心 不減, 大道可得。”孫氏道：“弟子悟一上乘, 豈肯悟中乘, 下乘？”王祖道：“方才 說過：你這容貌焉能悟上乘的功夫? 悟一個下乘也就罷了。”孫氏道：“師父，你說 我的容貌不能悟上乘的功夫, 這有何難? 略等片時, 在看我的容顏何如。”孫氏即 道廚房, 將油放在鍋內, 架起火來, 登時把烏雲燒盡; 面目放在油鍋裏邊, 著水點 煉個難看的模樣; 臉上刺破。走到師父面前, 叫聲：“師父, 弟子這個模樣, 可悟 上乘否?”王祖一看, 大吃一驚, 見孫氏合醜鬼一般, 說到: “善哉, 善哉世上那有 這樣狠心的女子? 真乃女中丈夫! 就是男子中也無這大剛大智之人! ”遂作詩一 首。詩曰：

女中丈夫悟上乘, 大剛大智可修行

火煉容貌似醜鬼, 功滿行完白日升。

王祖贊歎不盡, 說到：“你真吾弟子。起來，聽我吩咐：有這樣決烈, 何愁不到上 仙之位? 師父領天命道山東度人, 還有許多男女得了我的大道, 你定是第一名, 早 升天界。若到瓊瑤紫府, 靈霄殿上, 標名掛號。聽師父與你傳道, 莫有多言: 只要 把心腎扭轉, 搬轉斗柄, 運轉天星, 性命二字雙修, 盡矣。二六時中, 不可起念, 要隨起隨減。久日久之, 自然全無。日用功夫, 如同劍刎心腸, 制伏天機, 將地機 殺死。地下人心死, 上方一個仙。心若死了, 四肢身體自然得活。鎖住義馬, 拿住 
心猿, 要做神仙。功完行滿, 如此僅記, 不可泄漏。你如今去地道河南府洛陽縣, 那幾人與你有緣; 假裝風魔, 肚中飢了, 挨門乞食; 到晚投宿, 或在廊檐之下, 或 在破瓦窯中。功完行滿, 自有丹書下詔, 才能了當。為師與你取個法名, 叫做孫不 二。快去吧! ”孫氏拜謝道：“師徒今日分別, 幾時見面? ”王祖道：“只等你大道成 就, 在行外功八百, 仍舊到你家把你夫住點化提醒, 你我師徒在靈霄殿中見面。” 孫不二拜別師父, 瘋了魔了, 身穿破衣, 竟往河南洛陽縣。一路上討飯充飢, 夜住 曉行。不日來到洛陽縣, 宿住古廟裏邊, 白日上街乞食, 有一月天氣。街坊上做買 賣的人都說：“那裏來這個瘋女人??”眾人就問孫不二：“你從那裏來的? ”孫不二並 不回言, 只管胡說亂語。看他頭臉上, 好像火燒的孫猴子。眾人再不細問。 